\title{
Guest Editorial: Emerging Visual Information Processing Technologies for Multimedia Applications
}

\author{
Huanqiang Zeng ${ }^{1} \cdot$ Zhe Wei $^{2}$
}

Published online: 29 February 2016

(C) Springer Science+Business Media New York 2016

Visual information becomes an indispensable part of our life through diversified multimedia applications, such as smart phones, IPAD, high definition TV, three dimensional TV and free viewpoint $\mathrm{TV}$, to name a few. To provide more interactive and realistic multimedia experience, a new set of visual information processing technologies are emerging. These involve several research fields, such as visual information representation, compression, transmission and display technologies for various multimedia applications. The goal of this special issue is to present the latest research achievements of the emerging visual information processing technologies for multimedia applications. This special issue includes 16 papers, which will be briefly discussed as follows.

Visual object detection, extraction and analysis are very meaningful and have various applications. The paper entitled "Comic Storyboard Extraction via Edge Segment Analysis" (10.1007/s11042-015-2680-8) aims to decompose the comic image into several frames, which plays a key role in producing the digital comic documents suitable for mobile reading. The authors propose new validation rules to reject the false storyboard detections; therefore, their algorithm achieves much better performance than the existing methods. In touch screen manufacturing, the defect inspection is one of the most important processes. The paper entitled "Touch Screen Defect Inspection based on Sparse Representation in Low Resolution Images" (10.1007/s11042-015-2559-8) proposes a novel defect inspection approach based on sparse representation of low resolution image. Based on their simulation results, the proposed method achieves high inspection accuracy for different defect types. In the paper entitled "Hierarchical Salient Object Detection Model using Contrast-based Saliency and Color Spatial Distribution" (10.1007/s11042-015-2570-0), the authors aim to accurately interpret object saliency in image, in which the distinctive features are investigated from a global perspective. The effectiveness of this proposed model has been validated on MSRA database.

Huanqiang Zeng zeng0043@hqu.edu.cn

Zhe Wei

wei.zhe@huawei.com

1 School of Information Science and Engineering, Huaqiao University, Xiamen, China 361021

2 Huawei International Pte Ltd, Singapore, Singapore 
Visual enhancement, such as video restoration, denosing, is very essential to improve the visual fidelity. The paper entitled "Video Restoration Based on PatchMatch and Reweighted Low-rank Matrix Recovery" (10.1007/s11042-015-25451) presents a very interesting video restoration approach. By using a modified random PatchMacth algorithm, the authors find that the nearest-neighbour patches among the video frames can de-grouped quickly and accurately. The experimental results have shown that the proposed system achieves good performance in some image processing tasks, such as denosing and inpainting. The method for rain and snow removal from a single image has been investigated in the paper entitled "Single Image Rain and Snow Removal via Guided L0 Smoothing Filter" (10.1007/s11042-015-2657-7). The authors design a guided L0 smoothing filter to solve three relative optimization problems. In the paper entitled "Blind Image Noise Level Estimation using Texture-based Eigenvalue Analysis" (10.1007/s11042-015-2452-5), the authors present a method of blind image noise estimation. They utilize the eigenvalue analysis to mathematically derive a new noise level estimator based on the weak-textured image patches. The paper entitled "An Automatic Panoramic Image Mosaic Method Based on Graph Model" (10.1007/s11042-015-2619-0) presents a multi-image stitching method based on graph model. Experimental results have demonstrated the effectiveness in visual enhancement by the above proposed methods.

Perceptual visual information processing has attracted more and more attentions. The paper entitled "Bursty Interference-oriented Video Quality Assessment Method" (10.1007/s11042-015-2787-y) aims to assess video quality under bursty interference environment. The paper entitled "A Harmonic Means Pooling Strategy for Structural Similarity Index Measurement in Image Quality Assessment" (10.1007/s11042-0152620-7) proposes a novel pooling strategy based on harmonic mean to enhance the performance of the classical visual quality assessment-SSIM. In addition, the perceptual coding has been investigated in the paper entitled "Perceptual Ratedistortion Optimization for H.264/AVC Video Coding from Both Signal and Vision Perspectives" (10.1007/s11042-015-2533-5). The former two papers have shown their effectiveness and accuracy in perceptual quality assessment while the later one greatly improves the perceptual coding performance of H.264/AVC.

Error resilient ability is very important for visual information transmission. In "Layered Multiple Description Video Coding Using Dual-tree Discrete Wavelet Transform and H.264/AVC" (10.1007/s11042-015-2546-0), the authors propose an interesting HLMDVC framework to provide a robust and flexible video transmission under unreliable networks. Based on their simulation results, they have shown that the proposed framework is superior to some recently published state-of-the-art multiple description video coding methods.

Machining learning-based visual information processing methods are widely investigated. In the paper entitled "Data and Feature Mixed Ensemble Based Extreme Learning Machine for Medical Object Detection and Segmentation" (10.1007/ s11042-015-2582-9), the authors propose a new method of data and feature mixed ensemble based extreme learning machine (ELM). It can be seen from their results on various datasets that their method is robust and efficient for medical object detection and segmentation. In the paper entitled "Landmark Recognition with Compact BoW 
Histogram and Ensemble ELM" (10.1007/s11042-014-2424-1), the authors employ the discriminative feature selection method and an improved ELM algorithm. The experimental results have shown that the proposed algorithm achieves good performance in landmark recognition. In the paper entitled "Imaging and Representation Learning of Solar Radio Spectrums for Classification" (10.1007/ s11042-015-2528-2), the authors exploit the deep learning method for the representation learning of the solar radio spectrums. Experimental results have demonstrated the proposed network can effectively classify the solar radio images into the labelled categories.

This special issue also includes the study of some interesting visual information processing applications. In the paper entitled "A New Method to Estimate Ages of Facial Image for Large Database" (10.1007/s11042-015-2485-9), the authors aim to estimate the age of a facial image. They point out that the estimated ages should be a dynamic range rather than a specific one. The paper entitled "Pure Spatial Rich Model Features for Digital Image Steganalysis" (10.1007/s11042-015-2521-9) presents an effective steganalysis method, which uses statistics of neighbouring samples as features to capture the dependency changes caused by embedding. The experimental results of these methods are promising and comparable to the state-ofthe-art ones.

These 16 papers in this special issue cover a wide range of visual information processing technologies for multimedia applications. We hope that this special issue will be a good reference for those researchers who are devoting to this area, and also for those who wish to snapshot the current progress of this area. Finally, the guest editors would like to express the sincere thanks to all the reviewers for their valuable review comments, and the Editor-inChief-Prof. Borko Furht, and all the staffs of this journal for their great efforts in publishing this special issue.

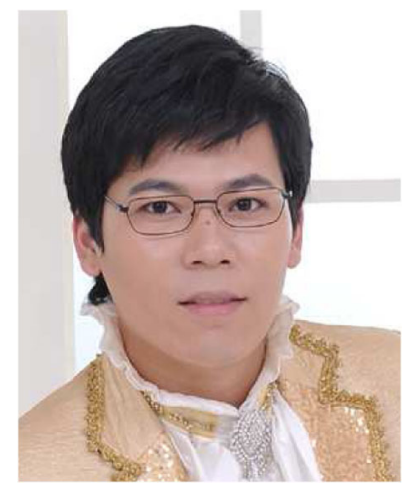

Huanqiang Zeng received the B.S. and M.S. degrees from Huaqiao University, Xiamen, China, and the Ph.D. degree from Nanyang Technological University, Singapore, all in electrical engineering. He was a Research Associate with Temasek Laboratories, Nanyang Technological University, Singapore and a Post-Doctoral Fellow with the Department of Electronic Engineering, The Chinese University of Hong Kong, Hong Kong. Currently, he is a Professor with the School of Information Science and Engineering, Huaqiao University, Xiamen, China. His research interests include visual information processing, video communication, 3-D/multiview video processing, and computer vision. 


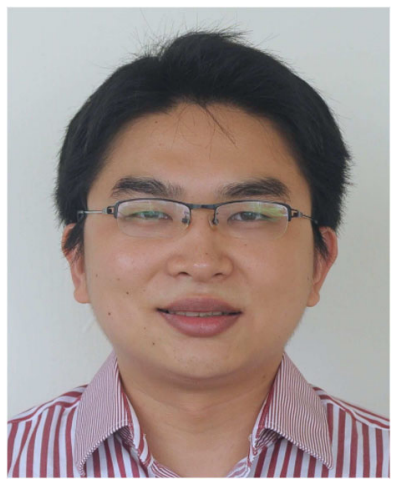

Zhe Wei received the B.S. and M.S. degrees from Huaqiao University, Xiamen, China, and the Ph.D. degree from Nanyang Technological University, Singapore, all in electrical engineering. He was a scientist with Temasek Laboratories, Nanyang Technological University, Singapore and a Post-Doctoral Fellow with the Department of Computer Engineering, McMaster University, Canada. Currently, he is a Senior Engineer with Huawei international Pte Ltd, Singapore. His research interests include image processing, image/video compression and communication, and computer vision. 